Supporting Information

\title{
Structural Insights from Tandem Mass Spectrometry, lon mobility-Mass Spectrometry and Infrared/Ultraviolet spectroscopy on sphingonodin I: Lasso vs. Branched-cyclic Topoisomers
}

Kevin Jeanne Dit Fouque, ${ }^{\dagger}$ Valeriu Scutelnic, ${ }^{\Perp}$ Julian D. Hegemann, ${ }^{\ddagger}$ Sylvie Rebuffat, ${ }^{\S}$ Philippe Maître, "Thomas R. Rizzo" and Francisco Fernandez-Lima.*,†

${ }^{\dagger}$ Department of Chemistry and Biochemistry, Florida International University, 11200 SW $8^{\text {th }}$ St., AHC4-233, Miami, FL 33199, United States.

" Laboratory of Molecular Physical Chemistry, Ecole Polytechnique Fedérale de Lausanne, Station 6, CH-1015 Lausanne, Switzerland.

₹ Department of Chemistry, University of Illinois at Urbana-Champaign, 6oo South Mathews Avenue, Urbana, IL 61801, United States.

$\S$ Laboratory Molecules of Communication and Adaptation of Microorganisms, National Museum of Natural History, CNRS, 57 rue Cuvier, CP-54, 75005 Paris, France.

\# Laboratoire de Chimie Physique, Université Paris Sud, UMR 8ooo CNRS, Faculté des Sciences, Bât. 349, 91405

Orsay Cedex, France.

Corresponding Author

fernandf@,fiu.edu

Table of Contents:

Figure S1. General classification criteria for lasso peptides.

Figure S2. TIMS-MS instrument showing the TIMS cell schematic and TIMS operation.

Figure S3. CID spectra of the doubly protonated species of (a) sphingonodin I and (b) its branched-cyclic topoisomer.

Table S1. Experimental vibrational frequencies $\left(\mathrm{cm}^{-1}\right)$ for the $[\mathrm{M}+2 \mathrm{H}]^{2+}$ ions of sphingonodin I and the branched-cyclic topoisomer in the $1350-1800$ and $2800-3700 \mathrm{~cm}^{-1}$ spectral regions. 

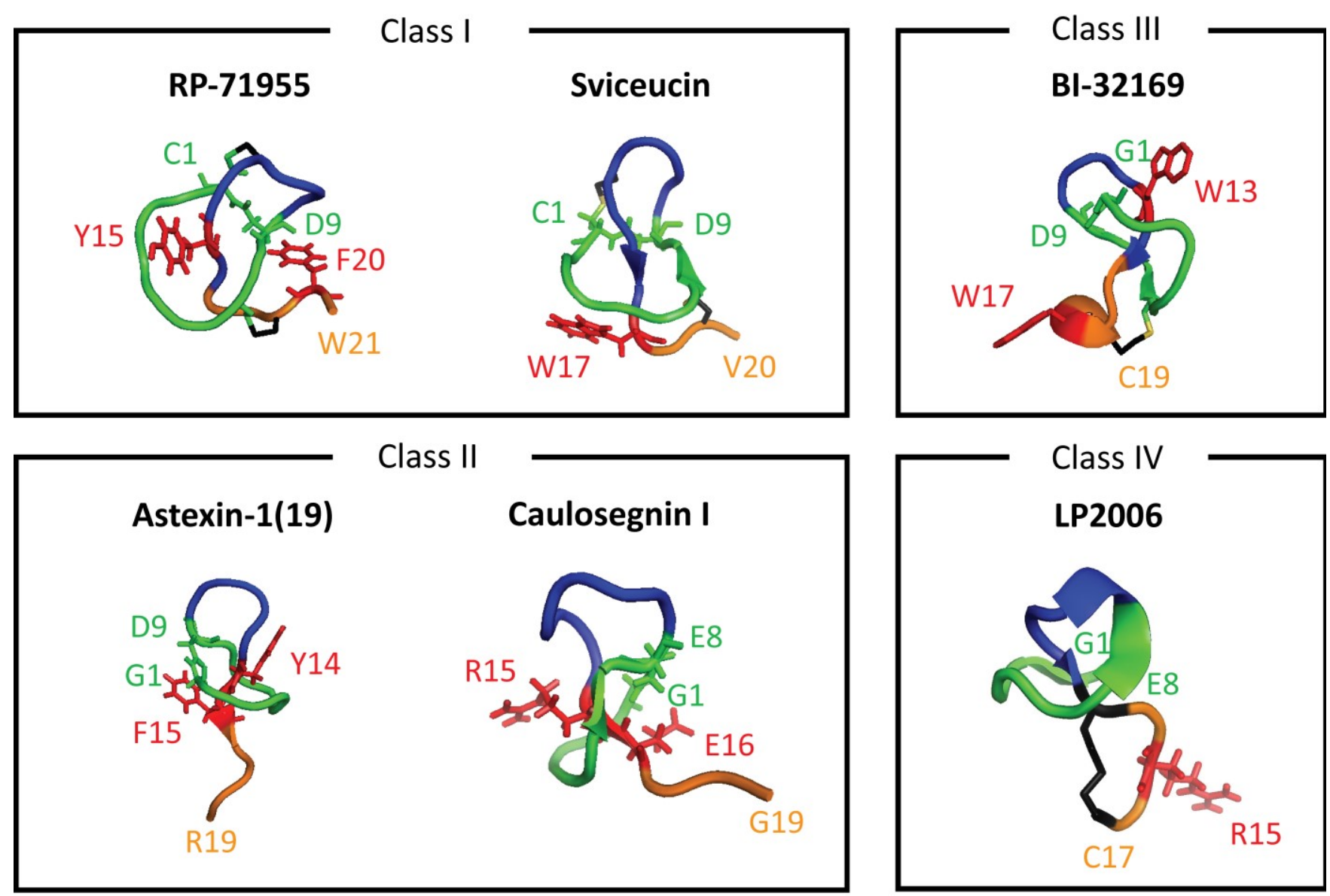

Figure S1. General classification criteria for lasso peptides. The lasso topology of RP-71955 (PDB 1 RPB) [1], sviceucin (PDB 2LS1) [2] (class I, two interlinked disulfide bonds), astexin-1(19) (PDB 2M37) [3], caulosegnin I (PDB 2LX6) [4] (class II, no disulfide bond), BI-32169 (PDB 3 NJW) [5] (class III, one interlinked disulfide bond) and LP2006 (PDB 5JPL) [6] (class IV, one handcuff disulfide bond) are shown. Tridimensional structures display the macrolactam rings in green, the loops in blue, the plugs in red and the C-terminal tails in orange. The disulfide bonds are shown by black lines. 


\section{Trapped Ion Mobility Spectrometry (TIMS)}

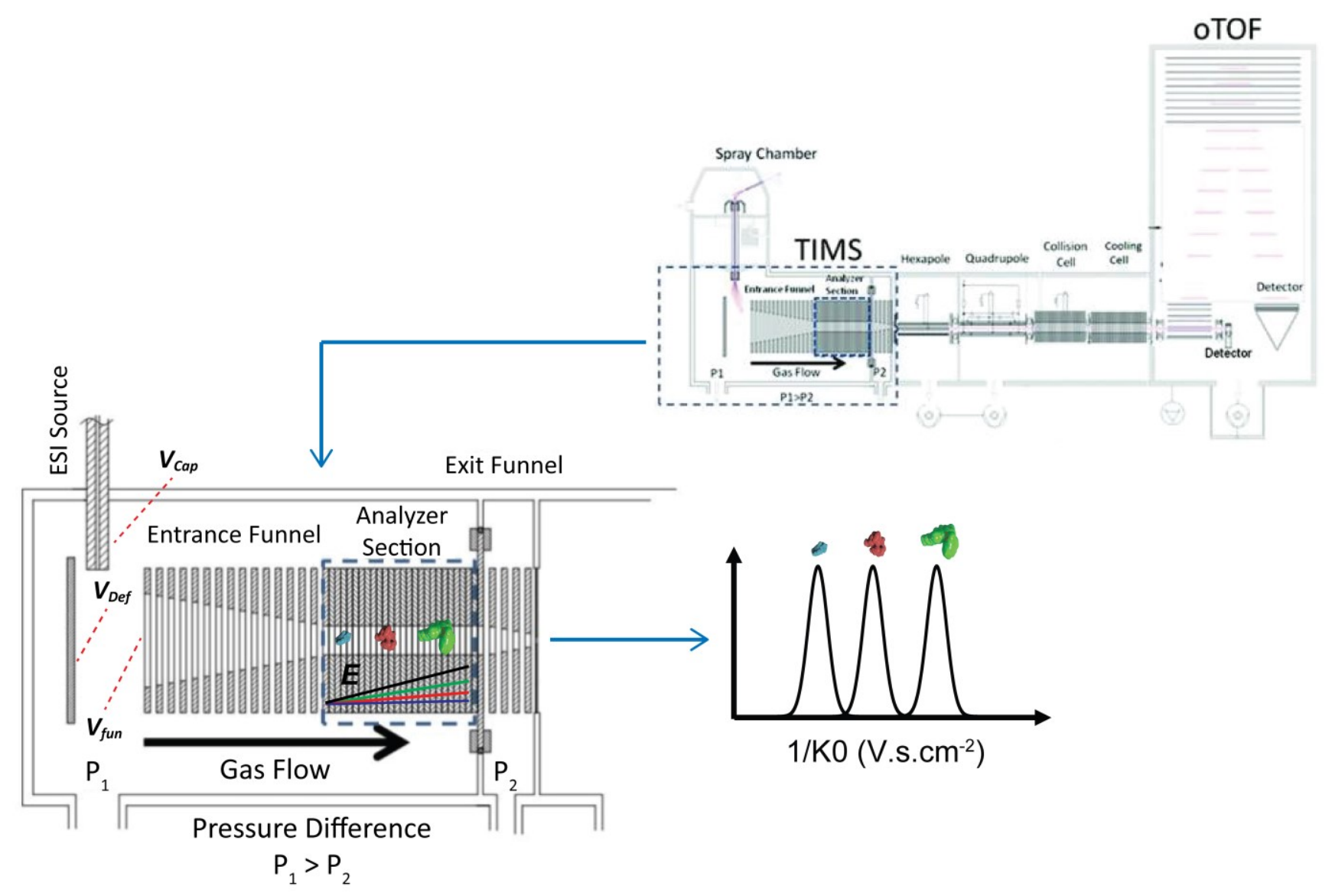

Figure S2. TIMS-MS instrument showing the TIMS cell schematic and TIMS operation. 


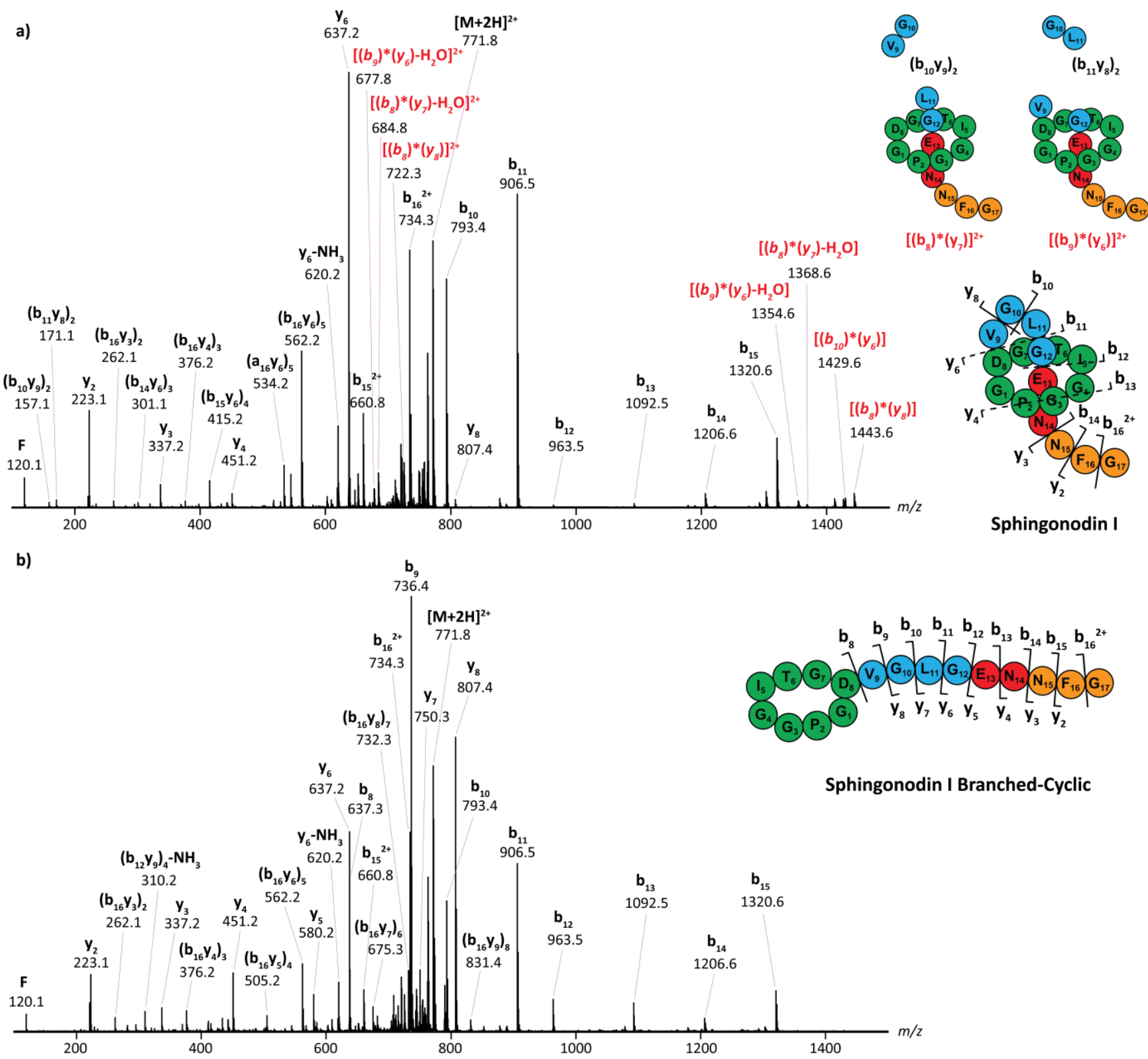

Figure $S_{3}$. CID spectra of the doubly protonated species of (a) sphingonodin I and (b) its branched-cyclic topoisomer $(\mathrm{m} / \mathrm{z}$ 771.8). Typical lasso-specific mechanically interlocked product ions are highlighted in red and labeled on the peptide cartoons (right of each panel). The macrolactam rings, the loop residues, the plugs and the C-terminal tails are highlighted in green, blue, red and orange, respectively. 
Table S1. Experimental vibrational frequencies $\left(\mathrm{cm}^{-1}\right)$ for the $[\mathrm{M}+2 \mathrm{H}]^{2+}$ ions of sphingonodin I and the branched-cyclic topoisomer in the $1350-1800$ and $2800-3700 \mathrm{~cm}^{-1}$ spectral regions. Differences between the two topoisomers are highlighted in red.

\begin{tabular}{|c|c|c|c|}
\hline \multirow{2}{*}{ Peptide } & \multicolumn{2}{|c|}{ IR vibrational frequencies $\left(\mathrm{cm}^{-1}\right)$} & \multirow{2}{*}{ Tentative assignment } \\
\hline & IR FEL & $\mathrm{OPO} / \mathrm{A}$ & \\
\hline \multirow{11}{*}{ Sphingonodin I } & $1410-1480$ & - & Aliphatic/Aromatic $\mathrm{CH}$ bend \\
\hline & 1535 & - & Amide NH bend \\
\hline & 1665 & - & H-bonded $\mathrm{C}=\mathrm{O}$ stretch \\
\hline & 1715 & - & Carbonyl $\mathrm{C}=\mathrm{O}$ stretch \\
\hline & - & $2925-3010$ & Aliphatic/Aromatic CH stretch \\
\hline & - & $3040-3130$ & H-bonded carboxylic acid OH stretch \\
\hline & - & $3240-3290$ & H-bonded alcohol OH stretch \\
\hline & - & $3300-3415$ & H-bonded amide $\mathrm{NH}$ stretch \\
\hline & - & 3440 & Amide $\mathrm{NH}_{2}$ sym. stretch \\
\hline & - & 3480 & Free amide NH stretch \\
\hline & - & 3540 & Amide $\mathrm{NH}_{2}$ asym. stretch \\
\hline \multirow{13}{*}{$\begin{array}{l}\text { Sphingonodin I } \\
\text { branched-cyclic }\end{array}$} & - & 3560 & Free carboxylic acid OH stretch \\
\hline & $1410-1480$ & - & Aliphatic/aromatic $\mathrm{CH}$ bend \\
\hline & 1570 & - & H-bonded amide $\mathrm{NH}$ bend \\
\hline & $1610-1670$ & - & H-bonded $\mathrm{C}=\mathrm{O}$ stretch \\
\hline & 1725 & - & Carbonyl $\mathrm{C}=\mathrm{O}$ stretch \\
\hline & - & $2925-3010$ & Aliphatic/Aromatic CH stretch \\
\hline & - & $3040-3130$ & H-bonded carboxylic acid OH stretch \\
\hline & - & $3215-3415$ & H-bonded amide $\mathrm{NH}$ stretch \\
\hline & - & 3440 & Amide $\mathrm{NH}_{2}$ sym. stretch \\
\hline & - & 3480 & Free amide NH stretch \\
\hline & - & 3540 & Amide $\mathrm{NH}_{2}$ asym. stretch \\
\hline & - & 3560 & Free carboxylic acid OH stretch \\
\hline & - & 3640 & Free alcohol OH stretch \\
\hline
\end{tabular}




\section{REFERENCES}

1. Frechet, D., Guitton, J.D., Herman, F., Faucher, D., Helynck, G., Monegier du Sorbier, B., Ridoux, J.P., James-Surcouf, E., Vuilhorgne, M.: Solution structure of RP 71955, a new 21 amino acid tricyclic peptide active against HIV-1 virus. Biochemistry. 33, 42-50 (1994)

2. Li, Y., Ducasse, R., Zirah, S., Blond, A., Goulard, C., Lescop, E., Giraud, C., Hartke, A., Guittet, E., Pernodet, J.L., Rebuffat, S.: Characterization of Sviceucin from Streptomyces Provides Insight into Enzyme Exchangeability and Disulfide Bond Formation in Lasso Peptides. ACS Chem. Biol. 10, 2641-2649 (2015)

3. Zimmermann, M., Hegemann, J.D., Xie, X., Marahiel, M.A.: The astexin-1 lasso peptides: biosynthesis, stability, and structural studies. Chem. Biol. 20, 558-569 (2013)

4. Hegemann, J.D., Zimmermann, M., Xie, X., Marahiel, M.A.: Caulosegnins I-III: a highly diverse group of lasso peptides derived from a single biosynthetic gene cluster. J. Am. Chem. Soc. 135, 210-222 (2013)

5. $\quad$ Knappe, T.A., Linne, U., Xie, X., Marahiel, M.A.: The glucagon receptor antagonist BI-32169 constitutes a new class of lasso peptides. FEBS Lett. 584, 785-789 (2010)

6. Tietz, J.I., Schwalen, C.J., Patel, P.S., Maxson, T., Blair, P.M., Tai, H.C., Zakai, U.I., Mitchell, D.A.: A new genome-mining tool redefines the lasso peptide biosynthetic landscape. Nat. Chem. Biol. 13, 470-478 (2017) 